\title{
Adsorption of Dodecanethiol on Cu(110): Structural Ordering upon Thiolate Formation
}

\author{
A. Kühnle, ${ }^{\dagger}$ S. Vollmer, ${ }^{\ddagger}$ T. R. Linderoth, ${ }^{\dagger}$ G. Witte, ${ }^{*, \neq}$ C. Wöll, ${ }^{\ddagger}$ and \\ F. Besenbacher ${ }^{\dagger}$
}

CAMP and Institute of Physics and Astronomy, University of Aarhaus, DK-8000, AarhausC, Denmark, and Lehrstuhl für Physikalische Chemiel, Ruhr-U niversität Bochum, D-44780 Bochum, Germany

Received February 25, 2002. In Final Form: May 7, 2002

\begin{abstract}
The adsorption of dodecanethiol $\left[\mathrm{CH}_{3}\left(\mathrm{CH}_{2}\right)_{11} \mathrm{SH}\right]$ films on $\mathrm{Cu}(110)$ by vapor deposition under ultrahigh vacuum conditions has been studied by means of thermal desorption spectroscopy, scanning tunneling microscopy, X-ray photoel ectron spectroscopy (XPS), and low-energy electron diffraction with a special emphasis on the structural changes accompanying the transition from a physisorbed monolayer to a chemisorbed saturation structure. Adsorption at $110 \mathrm{~K}$ leads to the formation of an ordered physisorbed layer with flat-lying thiol molecules. U pon room-temperature deposition, initially an ordered pinstripe phase is formed which may be a molecular double layer. This layer transforms with time into a stable saturation structure of upright-tilted thiolates in a local $c(2 \times 2)$ arrangement that exhibits a long-range $c(12 \times 16)$ modulation, attributed to a Moiré pattern. The XPS measurements show that the roomtemperature saturation structure contains a fraction of sulfide species formed by partial decomposition and desorption of al kyl chains. At $400 \mathrm{~K}$, thethiol atemonol ayer desorbs dissociatively, eventually resulting in a $p(5 \times 2)$ sulfur structure.
\end{abstract}

\section{Introduction}

Ultrathin molecular films, or self-assembled monolayers (SAMs), have attracted considerable interest, not least because of their promising technical applications in different fields such as lubrication, corrosion protection, high-resolution lithography, or biochemical surface functionalization. ${ }^{1}$ To date, the main body of data has been collected for monolayers of al kanethiols $\left[\mathrm{CH}_{3}\left(\mathrm{CH}_{2}\right)_{n-1} \mathrm{SH}\right]$ on $\mathrm{Au}(111)$, partly becausethis system is consi der ed to be prototypical for SAM formation, but also because such films are comparatively easy to prepare by immersion of a gold sample into a thiol solution. 2,3

Using an alternative preparation method, vapor-phase deposition, it has proven possible to trace the individual steps by which SAM s form in such systems, starting from mobile, physisorbed thiol molecules followed by dehydrogenation to chemisorbed thiolate species. ${ }^{4-6}$ Whereas no lateral ordering has been observed for the physisorbed alkanethiol mol ecules, a highly ordered "pinstripe" thiol ate phase, formed from molecules adsorbed with their backbones nearly parallel to the surface, has been identified at low coverages. With increasing coverage, several thiol ate phases are formed, accompanied by a continuous molecular upright tilting until a homogeneous and wellordered close-packed thiolatesaturation phaseis formed. ${ }^{7}$

\footnotetext{
* Corresponding author.

+ University of Aarhaus.

₹ Ruhr-Universität Bochum.

(1) Ulman, A. An Introduction to Ultrathin Organic Films: From Langmuir-Blodgett to Self-Assembly; Academic Press: Boston, 1991. UIman, A. Self-Assembled Monolayers of Thiols; Thin Films Vol. 24; Academic Press: San Diego, CA, 1998.

(2) Schreiber, F. Prog. Surf. Sci. 2000, 65, 151.

(3) Ulman, A. Chem. Rev. 1996, 96, 1533.

(4) Lavrich, D.J .; Wetterer, S. M.; Bernasek, S. L.; Scoles, G.J . Phys. Chem. B 1998, 102, 3456.

(5) Kondoh, H.; Kodama, C.; Sumida, H.; N ozoye, H.J . Chem. Phys. 1999, 111, 1175

(6) Schreiber, F.; Eberhardt, A.; Leung, T. Y. B.; Schwartzand, P.; Wetterer, A. M.; Lavrich, D. J .; Bermann, L.; Fenter, P.; Eisenberger,
} P.; Scoles, G. Phys. Rev. B 1998, 57, 12476.
This transition can bereversed by partial desorption from the saturated monolayer ${ }^{8-10}$

Compared to the case of gold, significant differences have been observed for alkanethiol films on other transition metals such as copper. For example, an adsorptioninduced reconstruction has been found for the $\mathrm{Cu}(111)$ surface, ${ }^{11}$ and a coexistence of thi olate and sulfide species has been identified in the saturated monolayer on $\mathrm{Cu}(100) .{ }^{12}$ Such observations demonstrate the need to extend the existing database on theordering mechanisms and properties of thiolate monolayers to a larger range of substrates.

Thecurrent pictureof theformation of alkanethiol SAMs on Cu surfaces follows that for Au with some deviations. Physisorbed al kanethiol molecules have been shown from $\mathrm{He}$ atom scattering to lie flat on copper surfaces, but no lateral ordering has been observed for these films. ${ }^{13} \mathrm{No}$ ordered low-coveragethiol ate phase with molecules lying prone on thesurface has hitherto been observed on copper. An ordered phase where the molecules are standing up with tilt angles between $12^{\circ}$ and $25^{\circ}$, al most independently of molecular chain length $(n=6-16)$, has been identified from X-ray absorption (NEXAFS, near-edge X-ray absorption fine structure) measurements on various low Miller index Cu surfaces. ${ }^{14-18}$ Annealing of such mono-

(7) Poirier, G. E.; Fitts, W. P.; White, J . M. Langmuir 2001, 17, 1176.

(8) Poirier, G. E.; Pylant, E. D. Science 1996, 272, 1145.

(9) Gerlach, R.; Polanski, G.; Rubahn, H. G. Appl. Phys. 1997, 65, 375.

(10) Staub, R.; Toerker, M.; Fritz, T.; Schmitz-Hübsch, T.; Sellam F.; Leo, K. Langmuir 1998, 14, 6693; Surf. Sci. 2000, 445, 100.

(11) Driver, S. M.; Woodruff, D. P. Langmuir 2000, 16, 6693.

(12) Vollmer, S.; Witte, G.; Wöll, C. Langmuir 2001, 17, 7560.

(13) Vollmer, S.; Fouquet, P.; Witte, G.; Boas, C.; Kunat, M.; Burghaus, U.; Wöll, C. Surf. Sci. 2000, 462, 135.

(14) Rieley, H.; Kendall, G. K.; Chan, A.; J ones, R. G.; Lüdecke, J .; Woodruff, D. P.; Cowie, B. C. C. Surf. Sci. 1997, 392, 143

(15) Imanishi, A.; I sawa, K.; Matsui, F.; Tsuduki, T.; Yokoyama, T.; Kondoh, H.; Kitajima, Y.; Ohta, T. Surf. Sci. 1998, 407, 282.

(16) Tsuduki, T.; Imanishi, A.; I sawa, K.; Terada, S.; Matsui, F.; Kiguchi, M.; Yokoyama, T.; Ohta, T. J . Synchrotron Rad. 1999, 6, 787. 
layers causes molecular dissociation with the sulfur remaining at the surface.

In this paper, we report on the adsorption of dodecanethiol $(n=12)$ on a Cu(110) surface, using thermal desorption spectroscopy (TDS), variable temperature scanning tunnelingmicroscopy (STM), X-ray photoelectron spectroscopy (XPS), and low-energy electron diffraction (LEED). The main focus of the present study is on the structural changes that accompany the transition from an initially physisorbed layer to a close-packed layer of dehydrogenated thiolate molecules. The study of this transition has, in part, been enabled by the right choice of a alkyl chain length for the al kanethiol molecule: since the molecular physisorption energy increases with the chain length, ${ }^{4}$ which is reflected by the temperatures for desorption and transition from physisorbed tochemisorbed states, respectively, the transition rate at room temperature is reduced for longer thiols and thus allows the detailed study of the intermediate phases. Previous structural studies were not able to investigate the physisorbed phase and the subsequent transition since they were performed at room temperaturefor short alkanethiols $(\mathrm{n}<\mathrm{8}) .11,19,20$

The main results from the present multitechnique investigation can besummarized as foll ows. At the lowest temperatureinvestigated, $110 \mathrm{~K}$, wefind an order ed layer of physisorbed mol ecules lying flat on the surface. At room temperature, a transition from a physisorbed to a chemisorbed state occurs. We observe two transient pinstripe structures which can be designated by $\left(\begin{array}{c}8-2 \\ 1\end{array}\right)$ and $c(2 \times 8)$ unit cells. Interestingly, indi cations exist that the former structure is a molecular double layer on top of the $c(2 \times$ 8) structure. Still at room temperature, these transient structures convert with time into a structure where the molecules are standing up, locally with an approximate $c(2 \times 2)$ arrangement, but exhibiting a long-range Moiré modulation resulting in a $\mathrm{c}(12 \times 16)$ unit cell. Within a few hours, or by heating briefly to $345 \mathrm{~K}$, a partial dissociation of this saturated thiolate film is observed, leading to the coexistence of sulfide and thiolate, still maintaining thec $(12 \times 16)$ Moirépattern. U pon annealing above $400 \mathrm{~K}$, dodecanethiol desorbs dissociatively, resulting in a $\mathrm{p}(5 \times 2)$ sulfur structure.

\section{Experimental Section}

The measurements reported here were performed in two different ultrahigh vacuum (UHV) systems. Thermal desorption spectroscopy, X-ray photoel ectron spectroscopy, and low-energy electron diffraction were performed in one system described in detail el sewhere. ${ }^{18}$ The STM measurements were obtained in another system, which is equipped with a home-built, variable temperaturescanning tunneling mi croscope ${ }^{21}$ as well as an Auger electron spectrometer and a mass spectrometer for residual gas analysis.

In both instruments, a $\mathrm{Cu}(110)$ sample was prepared by repeated cycles of $\mathrm{Ne}$ - or $\mathrm{Ar}$-ion sputtering foll owed by annealing above $800 \mathrm{~K}$ until a sharp LEED pattern with a low background signal was observed and theX-ray photoel ectron/Auger electron spectra or STM images revealed no traces of impurities. Dodecanethiol (Sigma Aldrich, 98\%), which had been purified thoroughly by freeze-pump-thaw cycles, was dosed onto the $\mathrm{Cu}$

(17) Gölzhäuser, A.; Panov, S.; Wöll, C. Surf. Sci. Lett. 1994, 314, L849.

(18) Loepp, G.; Vollmer, S.; Witte, G.; Wöll, C. Langmuir 1999, 15 3767. 207.

(19) Driver, S. M.; Woodruff, D. P. Surf. Sci. 2001, 479, 1; 2001, 488,

(20) Driver, S. M.; Woodruff, D. P. Surf. Sci. 2000, 457, 11.

(21) Petersen, L.; Schunack, M.; Schaefer, B.; Linderoth, T. R.; Rasmussen, P. B.; Sprunger, P. T.; Læegsgaard, E.; Stensgaard, I.; Besenbacher, F. Rev. Sci. Instrum. 2001, 72, 1438.

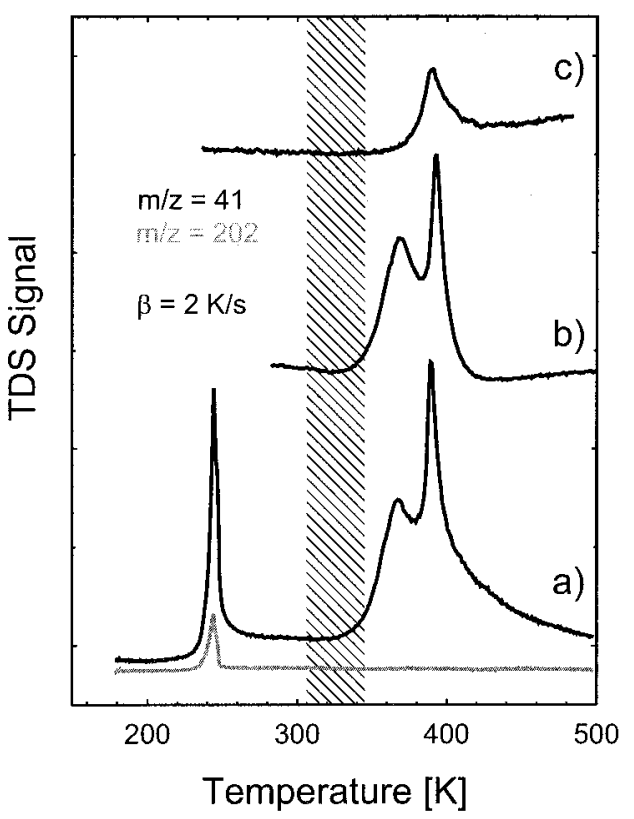

Figure 1. Series of thermal desorption spectra for dode canethiol adsorbed on $\mathrm{Cu}(110)$ recorded for the mass of the molecule ion ( $\mathrm{m} / \mathrm{z}=202$, gray line) and of the most prominent fragment $\mathrm{C}_{3} \mathrm{H}_{5}(\mathrm{~m} / \mathrm{z}=41$, black line) at a heating rate of $\beta=$ $2 \mathrm{~K} / \mathrm{s}$ : (a) for a multilayer film after exposure of 200 langmuir at $170 \mathrm{~K}$, (b) for a saturated monol ayer (250 langmuir) prepared at room temperature, and (c) a saturated monolayer after an additional annealing at $345 \mathrm{~K}$ for $10 \mathrm{~min}$. The shaded region around $340 \mathrm{~K}$ indicates the desorption temperature of physisorbed dodecanethiol estimated from an extrapolation of the corresponding desorption temperatures of shorter al kanethiols (see discussion section).

surfacethrough a leak valvefrom thevapor pressure of theliquid phase. The gas purity was monitored by mass spectrometry.

TheSTM images weretypically recorded with a negative bias of $-1.25 \mathrm{~V}$ applied to the sample and a tunneling current of around $0.6 \mathrm{nA}$. These parameters werefound to benondestructive since an influence from the scanning process on the thiol film was only observed if thetunneling current was increased to about $4 \mathrm{nA}$.

\section{Results}

TDS. To survey the adsorption states of dodecanethiol on $\mathrm{Cu}(110)$, we first describe results from TDS measure ments. Because of a high fragmentation probability of dodecanethiol upon ionization in the mass spectrometer, TD spectra were recorded simultaneously for the mass of the entire molecule $(m=202)$ and the $\mathrm{C}_{3} \mathrm{H}_{5}$ fragment ( $\mathrm{m}$ $=41$ ) which was found to be the most probable fragment in the dodecanethiol gas-phase mass spectrum.

Figure la shows a typical TD spectrum obtained after exposing the $\mathrm{Cu}(110)$ surface to 200 langmuir of dodecanethiol ( 1 langmuir $\equiv 1 \times 10^{-6}$ Torr s) at a sample temperature of $170 \mathrm{~K}$. Three different desorption peaks are observed at temperatures of 242,362 , and $392 \mathrm{~K}$. Whereas the low-temperature peak could be measured for themass of theentiremoleculeand the $\mathrm{C}_{3} \mathrm{H}_{5}$ fragment, the other two peaks appeared only for the fragment. A similar result has been observed previously for the desorption of heptanethiol from $\mathrm{Cu}(110)^{18}$ as well as for various alkanethiols on $\mathrm{Cu}(100) .{ }^{12}$ In analogy to those studies, the lower temperature peak is attributed to multilayer desorption while the other desorption peaks reflect the dissociative desorption of alkyl chains upon $\mathrm{S}-\mathrm{C}$ bond breaking, which is characteristic for alkanethiolatefilms on copper surfaces. ${ }^{12,18}$ Thepresence of a double 


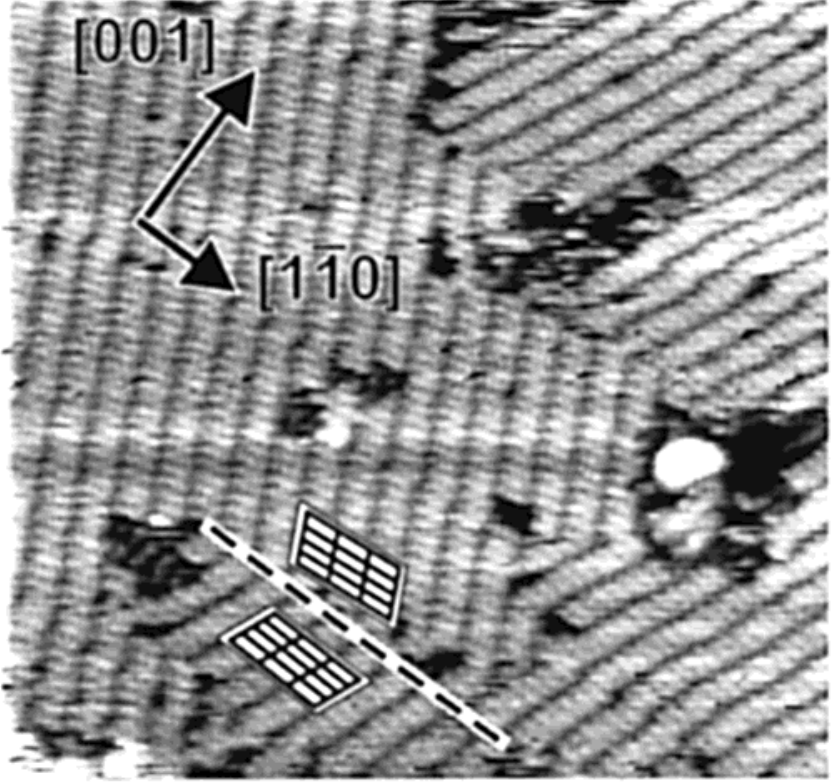

Figure 2. STM image $(283 \AA \times 274 \AA)$ recorded at $110 \mathrm{~K}$ after dodecanethiol adsorption at $110 \mathrm{~K}$. Two reflection domains of an ordered overlayer are seen.

peak is tentatively attributed to two slightly different adsorption geometries for the thiolates.

Activation energies of $63 \mathrm{~kJ} / \mathrm{mol}$ for desorption and 95 and $103 \mathrm{~kJ} / \mathrm{mol}$ for dissociation were calculated from the temperatures of the corresponding desorption peak maxima by applying the Redhead formula ${ }^{22}$ with a typical pre-exponential factor of $1 \times 10^{13} \mathrm{~s}^{-1}$.

To enable comparison with the STM data presented below, TD spectra weremeasured for two other adsorption conditions. First, TD spectra were recorded directly after saturating the surface with thiols at room temperature (Figure 1b), in which case the same high-temperature desorption doublet is observed. Second, TD spectra were recorded after annealing such a saturated monolayer for 10 min at $345 \mathrm{~K}$. As shown in Figure $1 \mathrm{c}$, this leads to a quenching of the lower temperature peak of the doublet while the desorption peak at $392 \mathrm{~K}$ remains.

STM. Low-Temperature Deposition. Upon deposition of dodecanethiol at $110 \mathrm{~K}$, an ordered overlayer is formed, as shown in the STM image in Figure 2. The overlayer structure consists of el ongated features approximately 6 $\AA$ wide and $17 \AA$ long, stacked in parallel to form rows. The size of these molecular features agrees well with the length of a dodecanethiol molecule (15.2 A), suggesting that the molecules are adsorbed parallel to the surface. Comparing STM images of this thiol structure to images of the bare surface, we find that the main axis of the molecules is rotated by $\pm 6^{\circ}$ with respect to the [1]10] direction of thesubstrate. Therotation leads totwo mirror domains as indicated by the grids superimposed in the STM image (see Figure 2).

Theexistenceof ordered domainsimplies that individual molecules are mobileat 110K. They diffuse on the surface until they areincor porated into the close-packed domains and become immobile. This picture is supported by the STM finding that the ordered domains have fuzzy edges, which weascribeto molecules attaching and detaching in equilibrium with a surrounding lattice-gas phase.

Room-Temperature Deposition. Next, results obtained by depositing and imaging the molecules at room temperature are presented. Upon gradually increasing the

(22) Redhead, P. A. Vacuum 1962, 12, 203.

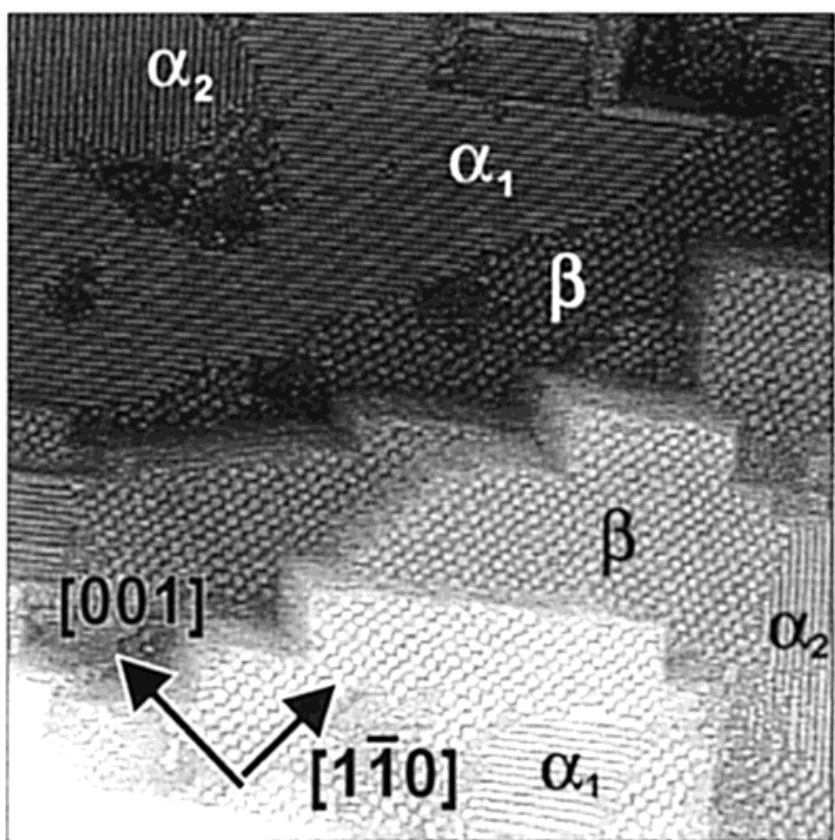

Figure 3. STM overview $(1830 \AA \times 1770 \AA)$ recorded at room temperature after dodecanethiol deposition at room temperature showing the coexistence of the transi ent $\alpha$ structure with two reflection domains $\left(\alpha_{1}, \alpha_{2}\right)$ and the $\beta$ structure.

exposure, clear evidence for adsorbed dodecanethiol was not observed until the surface was completely covered by an unorder ed mol ecular layer. This layer devel oped within afew minutes intotheordered structures discussed bel ow. We attribute the failure to observe dodecanethiol at submonolayer coverage to a high molecular mobility on the unsaturated surface at room temperature. This is further supported by the finding that the STM images recorded at low coverage show a high degree of noise and streakiness.

Figure 3 shows a large-scale STM image of the dodecanethiol monolayer after room-temperature saturation. Two coexisting structures (denoted $\alpha$ and $\beta$ ) can be identified, with the $\alpha$ phase existing as two reflection domains, $\alpha_{1}$ and $\alpha_{2}$.

A high-resolution STM image of the $\alpha_{2}$ structure is depicted in Figure $4 a$. Thestructure consists of el ongated entities arranged in rows to givethe characteristicstriped appearance seen in Figure 3. The periodicity along/ perpendicular to the stripes is 4.4 and $21.6 \AA$, respectively, and the stripes are oriented at angles of $\pm 55^{\circ}$ with respect tothe[110] di rection for thetwo reflection domains. In matrix notation, the $\alpha$ phase can be described by the $\left(\begin{array}{cc} \pm 8-2 \\ +1\end{array}\right)$ unit cell shown in Figure $4 a$. A height profile of the a structureis shown in Figure 4e, revealing a corrugation of about $0.9 \AA$. The STM images do not allow an unambiguous determination of the detailed molecular arrangement within this layer. However, to relate the structure of the $\alpha$ phase to the size of the dodecanethiol molecules, a possible molecular adsorption geometry, consisting of molecules adsorbed parallel to the surface with their sulfur atoms at the bright terminations of the elongated features, is indicated in the figure.

At some places, features were observed, as indicated by the arrow in Figure 4b, which appear to be holes in the $\alpha$ phase. As can be seen in the height profile depicted in Figure $4 \mathrm{f}$, these holes have a depth of around $0.7 \AA$. The structure found at the bottom of the holes, denoted $\gamma$, is depicted at high resolution in Figure 4c. The $\gamma$ structure exhibits periodicities of 5.1 and $28.9 \AA$ al ong the [1 $1 \overline{1} 0]$ and 

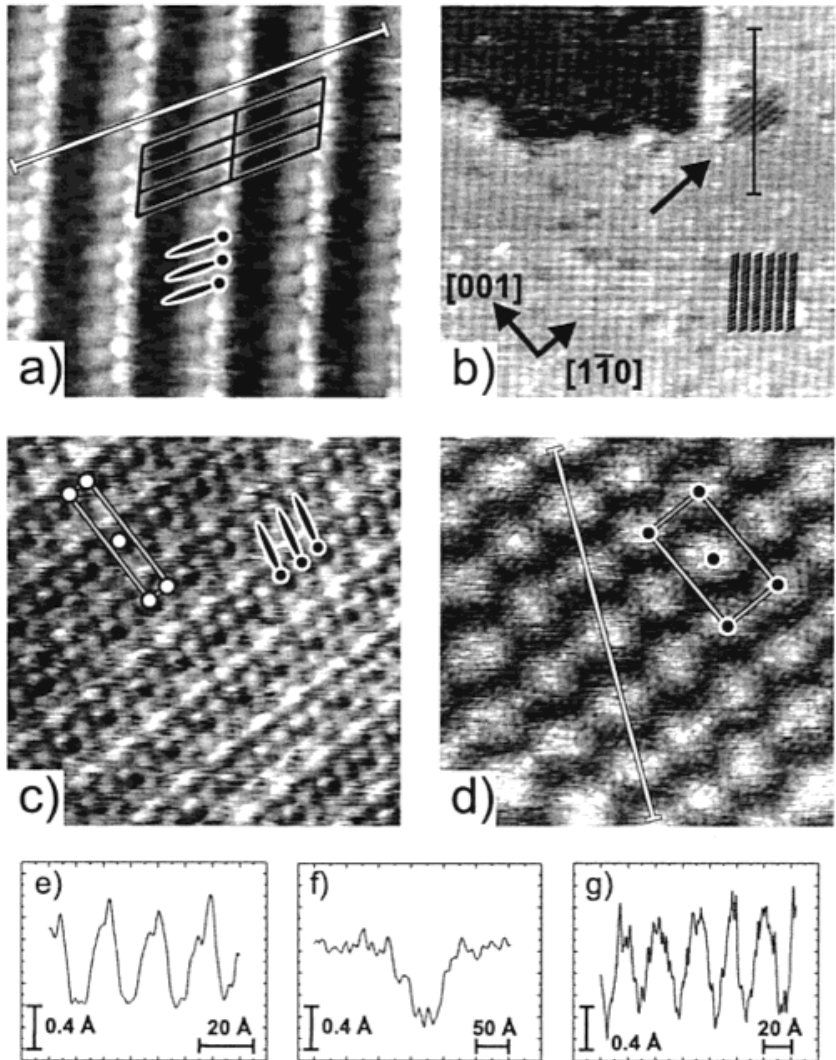

Figure 4. High-resolution STM images of the different dodecanethiol structures at room temperature. (a) I mage (85 $\AA \times 83 \AA$ ) showing one reflection domain of the transient $\alpha$ structure. A tentativestructuresuggestion is given by sketched lying down mol ecules. (b) I mage $(637 \AA \times 616 \AA)$ showing a hole in the $\alpha$ structure with the underlying $\gamma$ structure. (c) Zoom into the $\gamma$ structure $(122 \AA \times 118 \AA)$. One unit cell is indicated corresponding to a $\mathrm{c}(2 \times 8)$ structure. (d) $\beta$ structure exhibiting a $c(2 \times 2)$ structure with a $c(12 \times 16)$ Moiré pattern. $A c(12 \times$ 16) unit cell is marked $(187 \AA \times 177 \AA)$. (e) Height profile of the $\alpha$ structure at a position marked by the white line in (a). (f) Height profileover thehol eshown in (b). The position of this height profile is indicated by the black line in (b). (g) Height profile of the $\beta$ structure along the white line shown in (d).

[001] directions, respectively, corresponding to a $c(2 \times 8)$ unit cell as shown. A tentative suggestion for a molecular adsor ption geometry for the $\gamma$ structure is superposed on the STM image in Figure 4c. The observation of the $\gamma$ structure suggests the interesting possibility that the $\alpha$ phase described above might bea molecular doublelayer.

A zoom-in on the $\beta$ structure is shown in Figure $4 \mathrm{~d}$. This structure has a characteristic long-wavelength modulation with a corrugation amplitude of approximately $1.1 \AA$, as can be seen in the line scan in Figure $4 \mathrm{~g}$. The center-to-center distance between the protrusions of this long-range pattern is 31 and $58 \AA$ along the [1]10] and [001] directions, respectively, corresponding to a c(12 $\times$ 16) structure(indicated in thefigure). By zooming further in on this $\beta$ structure, a molecular-level fine structure is revealed which appears consistent with a local $c(2 \times 2)$ arrangement of theadsorbed species (a comparatively high noise level in these images prevents direct, unequivocal identification of long-range $c(2 \times 2)$ order in this layer $)$. We attribute the $\beta$ structure to molecules in an upright geometry, and as will be discussed in more detail below, the $c(12 \times 16)$ modulation is ascribed to a Moiré pattern resulting from a mismatch between the overlayer of approximate $c(2 \times 2)$ structure and the underlying substrate.

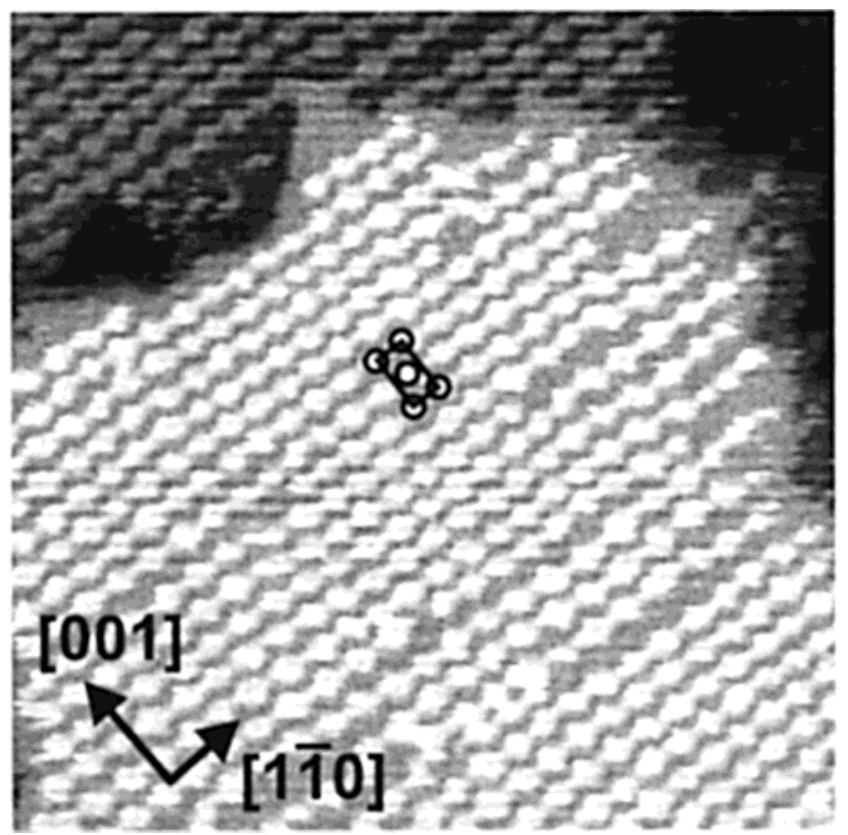

Figure 5. STM image of the saturation structure $(845 \AA \times 826$ A) after annealing the $\beta$ phase at $345 \mathrm{~K}$ for $10 \mathrm{~min}$ resulting in a slightly modified $\beta^{\prime}$ structure.

To directly investigate the evolution with time of the molecular overlayer structures, a series of consecutive STM images wereacquired of the samearea on thesurface (STM movies). By following the area depicted in Figure 3 for $130 \mathrm{~min}$ at room temperature, it was found that the two reflection domains of the $\alpha$ structureconvert reversibly into each other. Moreinterestingly, both $\alpha$ structures could be seen to convert into the $\beta$ structure. This eventually resulted in the $\beta$ phase covering the entire surface after about $2 \mathrm{~h}$, demonstrating that this phase is the energetically most favorable. However, the reverse $\beta$ to $\alpha$ conversion was also observed occasionally at the boundaries between these phases demonstrating local reversibility of the phase transition.

If thesaturated monolayer formed at roomtemperature is annealed gently at 345K for $10 \mathrm{~min}$, thestructureshown in F igure 5 is formed. This $\beta^{\prime}$ structure differs only slightly from the $\beta$ structure observed at room temperature and still reveals thelong-range order associated with thec(12 $\times$ 16) Moiré pattern. A probable reason for the different appearances of the two structures might be the onset of a partial dissociative desorption, as evidenced by the above-mentioned TDS results and XPS measurements discussed in the following.

When the molecular film is annealed above $420 \mathrm{~K}$, the molecules desorb dissociatively resulting in a $\mathrm{p}(5 \times 2)$ sulfur structure. ${ }^{23}$

Finally, we note that evaporation of dodecanethiol at room temperature introduces step faceting, changing the steps of the clean $\mathrm{Cu}(110)$ surface into the rectangular step features noticeable in Figure 3 . This effect becomes even more pronounced when the $\beta$ structure is fully devel oped. This step faceting is evidence for a substantial mobility of the substrateatoms at room temperatureand/ or possible adsorbate-mediated substrate modifications.

XPS.Toidentify and characterizethe different chemical adsorption states, temperature-dependent XPS measurements were carried out. Figure 6 shows a comparison of $\mathrm{S} 2 \mathrm{p}$ and $\mathrm{C}$ 1s XP spectra for dodecanethiol films on the

(23) Stensgaard, I .; Ruan, L.; Besenbacher, F .; ensen, F .; L æogsgaard, E. Surf. Sci. 1992, 269/270, 81. 


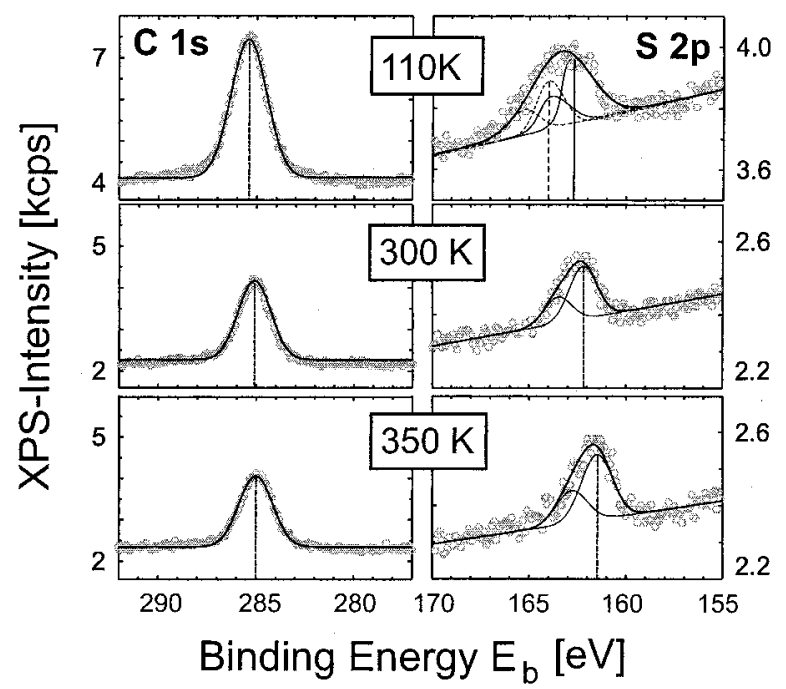

Figure 6. Series of $X P$ spectra of the $S 2 p_{3 / 2}$ and $C$ 1s peaks for dodecanethiol films prepared at $110 \mathrm{~K}$ (about 10 langmuir) and for saturation dosage (200 langmuir) at 300 and $350 \mathrm{~K}$. The data (gray circles) have been fitted by a set of one and two Gaussian curves for carbon and sulfur, respectively. The two sulfur doublets at low temperature can be attributed to a physisorbed monolayer (solid line) and thiol multilayers (dashed line).

$\mathrm{Cu}(110)$ surface taken after an exposure of about 10 langmuir at $110 \mathrm{~K}$ and after saturating the surface at 300 and $350 \mathrm{~K}$, respectively. All spectra have been referenced to a $\mathrm{Cu} 2 \mathrm{p}_{3 / 2}$ line energy of $932.4 \mathrm{eV} .{ }^{24}$ To determine the exact peak positions, the $52 \mathrm{p}$ doublets were fitted by two Gaussian curves with a fixed intensity ratio of $I_{3 / 2} / I_{1 / 2}=$ 2 and a fixed energy separation of $1.3 \mathrm{eV}$. Whiletheposition of the carbon peak remains almost constant at $285 \mathrm{eV}, \mathrm{a}$ temperature-dependent shift of the $S 2 p_{3 / 2}$ binding energy was observed. A fit to the room-temperature data yiel ded a S $2 p_{3 / 2}$ binding energy of $E_{B}=162 \mathrm{eV}$. By comparison, the sulfur peak observed for the low-temperature preparation is substantially broader and can bewell decomposed into two $S 2 p$ doublets with $S 2 p_{3 / 2}$ binding energies of 162.8 and $164 \mathrm{eV}$. A very similar result has been observed recently in a high-resolution XPS study of the adsorption of butanethiol on $\mathrm{Cu}(110)$ by employing synchrotron radiation. ${ }^{26}$ There, a physisorbed thiol monolayer and thiol multilayers could be clearly distinguished and corresponding S $2 p_{3 / 2}$ binding energies of 162.6 and $164.0 \mathrm{eV}$, respectively, have been determined. Thus, the present low-temperature measurements indicate a coexistence of a physisorbed monolayer and thiol multilayer. Moreover, thedistinct reduction of thesulfur binding energy by about $1 \mathrm{eV}$ when going to room temperature has been obtained before also for other cases of alkanethiols on copper surfaces and reflects the thiolate formation upon deprotonation of the sulfur headgroup. $12,18,25$

The analysis of the corresponding peak intensities, determined by integration of the fitted Gaussians after a linear background subtraction, yielded a $\mathrm{C} 1 \mathrm{~s} / \mathrm{S} 2 \mathrm{p}_{3 / 2}$ intensity ratio of $12.6 \pm 1$ for $110 \mathrm{~K}$, while it increases to $16.2 \pm 1$ for a film preparation at $300 \mathrm{~K}$. This change is attributed to an enhanced attenuation of the S $2 p$ line through the upright-tilted alkyl chains ${ }^{14-16}$ of the saturated thiolate phasecompared totheflat-lying physisorbed alkanethiol molecules on copper. ${ }^{13}$

(24) Wagner, C. D.; Riggs, W. M.; Davis, L. E.; Moulder, J. F. In Handbook of X-ray photoel ectron spectroscopy; Muillenberg, G. E., Ed.; Perkin-Elmer Corp.: Eden Prairie, MN, 1979.

(25) Kariapper, M. S.; Fischer, C.; Woodruff, D. P.; Cowie, B. C. C.; J ones, R. G. J . Phys.: Condens. Matter 2000, 12, 2153.

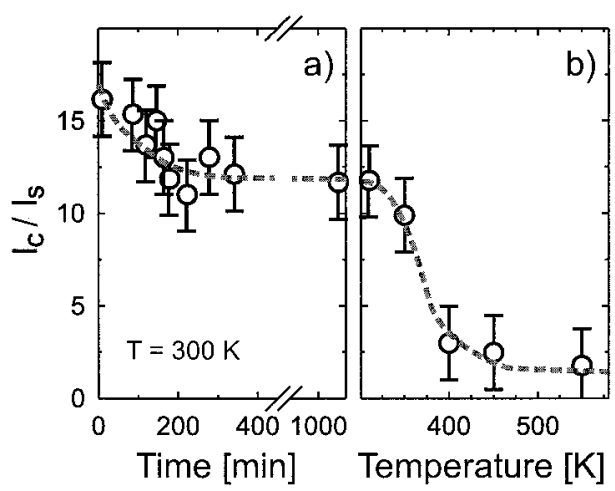

Figure 7. Evolution of the intensity ratio of the $C$ is and $S$ $2 p_{3 / 2} \times P$ peaks for dodecanethiol adsorbed at room temperature as a function of (a) time and (b) the subsequent annealing temperature. The data of (b) were all recorded at room temperature after the sample was held at the displayed annealing temperatures for $1 \mathrm{~min}$.

By following theXPS intensities with time, thestability of the saturated monolayer films could be followed. As shown in Figure $7 \mathrm{a}$, the $\mathrm{C} 1 \mathrm{~s} / \mathrm{S} 2 \mathrm{p}_{3 / 2}$ intensity ratio decreases froman initial value of $16.2 \pm 1$ after preparation to about $12 \pm 1$ within 200 min and subsequently remains constant at this level for a further $800 \mathrm{~min}$. A similar reduction of the $C 1 s / S 2 p_{3 / 2}$ intensity ratio was observed upon annealing of the thiolatefilm briefly to $350 \mathrm{~K}$. Since thecl ose packing of the saturated thiolatemonolayer does not allow the molecules to tilt downward again, this decrease in theratio is explained by a partial degradation of the dodecanethiol film causing the carbon intensity to decrease while the sulfur coverage remains essentially constant. We estimate that the alkyl chain density is reduced by about $20-25 \%$ at room temperature after equilibration.

A similar changein intensities was observed previously for various alkanethiol films on $\mathrm{Cu}(100),{ }^{12}$ where it was shown that the partial deterioration was not caused by radiation-induced damages. Toexcluderadiation damages in thepresent experiment, the recording timefor each XP spectrum was limited toabout $10 \mathrm{~min}$ and theX-ray source was switched off between the different time-resolved measurements.

A small shift of the sulfur $S 2 p_{3 / 2}$ binding energy to $E_{B}$ $=161.5 \mathrm{eV}$ accompanies the decrease in the $\mathrm{C} 1 \mathrm{~s} / \mathrm{S} 2 \mathrm{p}_{3 / 2}$ intensity ratio upon annealing to $345 \mathrm{~K}$ (see Figure 6). Unfortunately, the resolution in the present experiments was not sufficient to directly resolvetheantici pated sulfide species in the XPS data. We note, however, that the presence of such sulfide species has been clearly identified in a recent high-resolution XPS experiment by using synchrotron radiation. ${ }^{26}$

Upon additional annealing at elevated temperatures, a further decrease of the $C 1 s / S 2 p_{3 / 2}$ intensity ratio was observed as shown in Figure $7 \mathrm{~b}$. The corresponding XP spectra were all recorded at room temperature after the sample was held at the indicated temperature for about $1 \mathrm{~min}$. Theintensity ratiostarts to decreaseal ready above room temperature and vanishes al most completely after heating to $400 \mathrm{~K}$. This reflects that at temper atures above $400 \mathrm{~K}$, complete dissociativedesorption of all al kyl chains occurs with the sulfur remaining on the copper surface, in good agreement with the conclusions from the TDS and STM measurements.

LEED. Tofurther characterizethelong-rangeordering of the dodecanethiol films, low-energy el ectron diffraction

(26) Vollmer, S.; Witte, G.; Wöll, C. To be published 

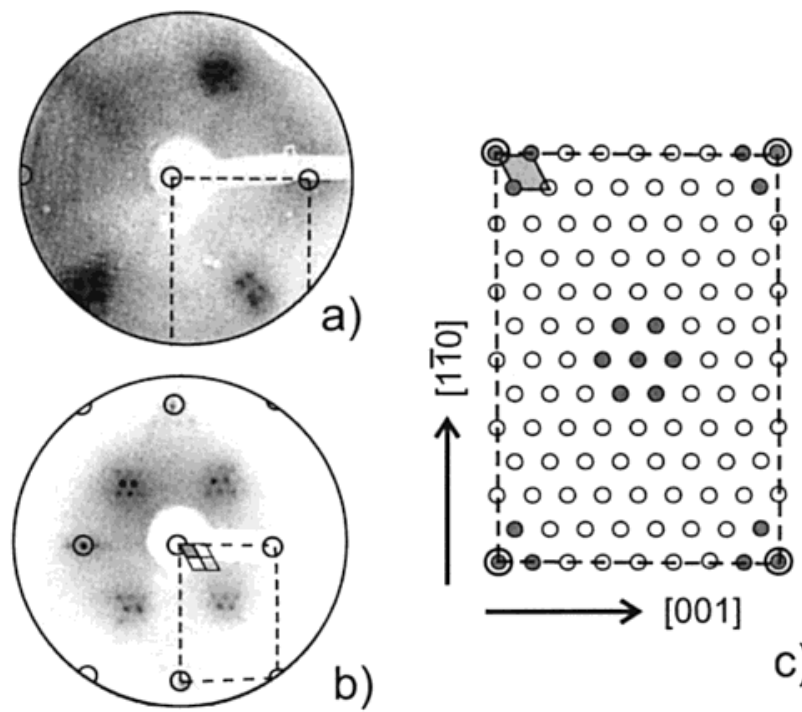

c)

Figure 8. (a) LEED pattern for a room-temperaturesaturation structure of dodecanethiol on the $\mathrm{Cu}(110)$ surface taken at an incident energy of $27 \mathrm{eV}$. F or comparison, a LEED pattern $\left(E_{i}\right.$ $=73 \mathrm{eV}$ ) for a saturated ethanethiol ate monolayer on $\mathrm{Cu}(110)$ is displayed in (b). Both diffraction patterns were recorded at $\mathrm{T}_{\mathrm{S}}=120 \mathrm{~K}$. F or clarification, the diffraction pattern of a c(12 $\times 16)$ structure is shown in (c) together with the unit cells of the superstructure (gray parallelogram) and the substrate (dashed rectangle). The visible diffraction spots (gray circles) are located near the $\mathrm{c}(2 \times 2)$ positions.

measurements were carried out. To prevent possible damage to the film from the electron beam, all LEED measurements wereperformed with a microchannel plate LEED system operated with a typical incident electron beam current of $100 \mathrm{pA}$. In view of the soft external vibrational modes of thin organic films, ${ }^{13,27}$ which lead to a strong Debye-Waller attenuation of the diffraction peaks, the sample was cooled to below $120 \mathrm{~K}$ after preparation beforerecording the LEED pattern. Attempts to observe any diffraction pattern for a physisorbed dodecanethiol layer prepared by exposing thesampleheld at $120 \mathrm{~K}$ were not successful. Instead, only an enhanced diffuse background signal was obtained.

After saturating the $\mathrm{Cu}(110)$ surfacewith dodecanethiol at room temperature, rather broad diffraction peaks at $c(2 \times 2)$ positions were obtained accompanied by an enhanced diffuse background as displayed in Figure 8a. Although these spots clearly reveal an internal intensity modulation, analysis of the underlying superstructure is hampered by a weak contrast. Previous studies have shown that saturation thiolate structures on various copper surfaces do not depend on the chain length $11,12,20$ while the contrast of the corresponding LEED patterns decreases with increasing chain length due to an attenuation of the diffraction intensity by the chains itself. ${ }^{12}$ Inspired by these observations, and in order to facilitate a more detailed structureanalysis, additional LEED data were also recorded for saturated monolayers of shorter alkanethiols with chain lengths ranging from $n=2-7$ on the $\mathrm{Cu}(110)$ surface. F igure8b displays the corresponding LEED pattern for ethanethiol, which has a structure identical to those obtained for the other chain lengths. It clearly reveals a narrow mesh of diffraction spots, as depicted schematically in Figure 8c, in agreement with a $c(12 \times 16)$ superstructure. Since diffraction spots were only observed near the $c(2 \times 2)$ positions, these data can beinterpreted in terms of a M oirépattern consistinglocally

(27) Witte, G.; Wöll, C. J . Chem. Phys 1995, 103, 5860. of a $c(2 \times 2)$ structure with a slight lattice mismatch forming the large $c(12 \times 16)$ unit cell as shown schematically in Figure 8c. The LEED data for the ethanethiolate monolayer is thus consistent with the large-scale periodicity observed in the STM images for the saturated dodecanethiol layer.

Returning to the LEED data for dodecanethiol, we note that no diffraction pattern was found corresponding to the intermediate pinstripe phases observed in the STM measurements. After annealing of the dodecanethiol film (as well as the shorter alkanethiol films) above $400 \mathrm{~K}$, a sharp $(5 \times 2)$ LEED pattern was observed, in agreement with the STM observation of such a sulfur structure. ${ }^{23}$

\section{Discussion}

In the following discussion, the different observations from the present multitechniquestudy will beinterrelated and compared to the existing literature in order to form a coherent picture of the adsorption of dodecanethiol on $\mathrm{Cu}(110)$.

Adsorption of dodecanethiol at low temperatures (110 K) leads to the formation of a physisorbed layer with an ordered pinstripe structure exhibiting two reflection domains (see Figure 2). The STM data suggest an orientation of the molecules parallel to the surface which is corroborated by the $C 1 s / S 2 p_{3 / 2}$ intensity ratio in the corresponding XPS measurements. The same molecular orientation has been derived from an analysis of the lowenergy vibrational modes for heptanethiol physisorbed on $\mathrm{Cu}(110){ }^{13}$

It is interesting to comparethis physisorbed phasewith the monolayer structure adopted by saturated hydrocarbon chains on the same surface. F or octane on $\mathrm{Cu}(110)$, a $\mathrm{p}(5 \times 6)$ monolayer structurehas been identified in which the alkane molecules are aligned with the close-packed [110] direction of the anisotropic surface. ${ }^{28} \mathrm{I}$ n the present low-temperature dodecanethiol film, the molecules are rotated by $\pm 6^{\circ}$ with respect to the close-packed direction, demonstrating the important influence of the endgroups on the resulting film structure.

Thelow-temperaturephysisorption structure could not beobserved by LEED. Weattributethis tothewidespread of diffraction spots due to the large unit cell size together with the domain structure and the small scattering cross section of S and C compared to the substrate which in combination result in weak diffraction spots (especially at out-of-phase conditions with respect to the substrate).

Theobserved shift of theXP S $2 p_{3 / 2}$ peak binding energy fromabout $163 \mathrm{eV}$ at $110 \mathrm{~K}$ to $162 \mathrm{eV}$ at room temperature indicates that full or partial conversion from physisorbed molecules to thi olates has occurred at the latter temperature. The expected desorption temperature for physisorbed dodecanethiol molecules can be obtained from extrapolation of the corresponding desorption temperatures obtained for shorter al kanethiols. ${ }^{26}$ This is possible because the physisorption energy scales almost linearly with the chain length due to the molecular adsorption geometry parallel to the surface. ${ }^{4}$ By this approach, we estimate the expected desorption temperature for physisorbed dodecanethiol to be $320 \pm 10 \mathrm{~K}$. This temperature band is indicated by the shaded region in Figure 1 but does not coincide with any desorption peaks. In fact, the TDS signal for the mass of the entire molecule shows no spectral features above the multilayer desorpti on peak at about $240 \mathrm{~K}$. In combination with the XPS results, this demonstrates that a complete conversion of the dodecanethiol molecules into chemisorbed thiolate species 
occurs during the thermal ramp of the desorption measurements. On the other hand, the chemical transition rate is reduced at room temperature where typical conversion times of up to several hours were found from the time-resolved XPS data of F igure 7a. This enabled the observation by room-temperature STM of several intermediate structures appearing during the formation of a saturated thiolate layer.

U pon evaporation of dodecanethiol at room temperature, initially a substantial surface mobility was inferred from the inability to perform STM imaging at submonolayer coverages. Again, this suggests that the individual molecules are initially adsorbed in a physisorbed precursor state which only becomes observable with STM once the molecules form a continuous island or film.

The first ordered structure formed upon room-temperature deposition is the pinstripe $\alpha$ phase with a $\left(\begin{array}{ll}8 & -2 \\ 1 & 1\end{array}\right)$ unit cell. With time, this structure converts into the $\beta$ phase characterized by local approximate $c(2 \times 2)$ order and a long wavelength Moiré modulation described by a c(12 $\times$ 16) unit cell.

It may seem tempting to equate the physisorption to chemisorption transition observed with XPS and TDS with the structural $\alpha$ to $\beta$ phase transition observed by STM. However, sincea hydrogen atom is lost upon deprotonation to form chemisorbed thiolatespecies, thelocal reversibility of this phase transition, as seen in the STM movies at the boundaries between the two phases, strongly suggests that the molecular species in both the $\alpha$ and $\beta$ phases are in the same, chemisorbed, state. ${ }^{29}$

Thesaturated monolayer of the $\beta$ structureis thermally metastable. Gentle annealing of the film at $345 \mathrm{~K}$ leads to a partial, dissociative desorption of the alkyl chains and causes a reduction of thefilm density. Thisis evidenced by the observed decrease of the $\mathrm{C} 1 \mathrm{~s} / \mathrm{S} 2 \mathrm{p}_{3 / 2}$ intensity ratio in the XPS data. Inter estingly, the resulting $\beta^{\prime}$ structure has essentially the same lateral structure as the $\beta$ structure, as seen by STM. The time-lapsed XPS measurements shown in Figure 7a indicate that a similar partial degradation of thefilm takes place on a timescale of several hours at room temperature. This demonstrates that the stable room-temperature saturation phase comprises coexisting thiolate and sulfide species. Such an instability has been observed previously for theadsorption of alkanethiols of various chain lengths on $\mathrm{Cu}(100)^{12}$ and thus seems to be quite general for the adsorption of thiolates on copper surfaces.

Heating the saturated film above $400 \mathrm{~K}$ causes a complete dissociative desorption of the alkyl chains, as observed by XPS and TDS, and leads to the formation of locally ordered domains of a $\mathrm{p}(5 \times 2)$ sulfur structure identical to that which has been observed before upon deposition of sulfur on $\mathrm{Cu}(110){ }^{23}$

We now discuss the $\beta$ structure in more detail. In general, theformation of a Moirépattern can beexplained by a coincidence mesh between two layers with a slight latticemismatch. In the present case, this could berealized either by a periodic substrate relaxation or by slight expansion/compression of the molecular overlayer away from a commensurate $c(2 \times 2)$ structure (as shown schematically in Figure 9a,b). Although the appearance of surfacestress upon chemisorption of mol ecules has been

(29) Rehydrogenation of the molecules in the $\beta$ phase to allow a transition from chemisorbed to physisorbed molecules seems unlikely as the strong $\mathrm{S}-\mathrm{Cu}$ interaction does not favor reformation of the comparatively weak $\mathrm{S}-\mathrm{H}$ bond. In the present TDS studies, hydrogen desorption was not monitored, since previous investigations by us have shown that the detection of a hydrogen desorption peak is hampered by a high hydrogen background signal.

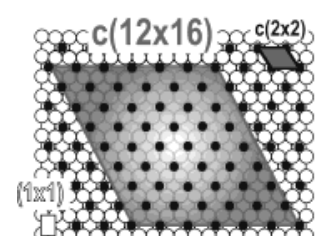

a)
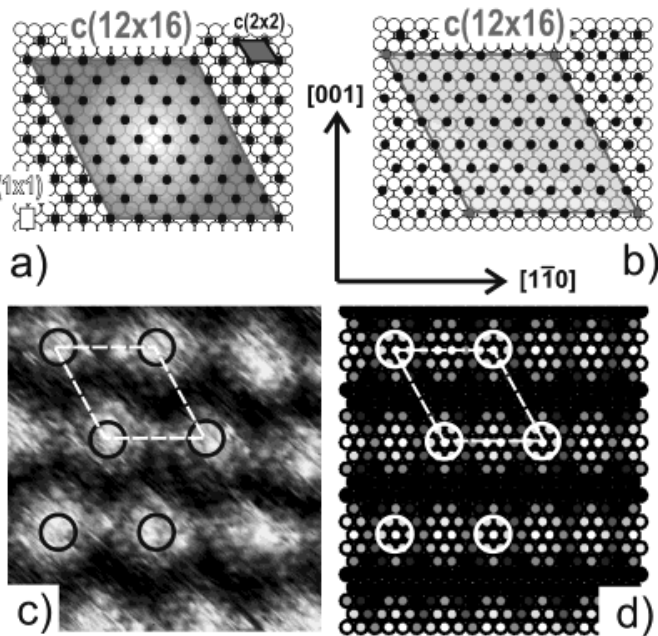

Figure 9. Panels $a$ and $b$ illustrate two possible reasons for the appearance of the $c(12 \times 16)$ Moiré pattern: (a) a $c(2 \times 2)$ phase with an additional modulation of the substrate (large parallelogram) due to a stress release pattern and (b) an anisotropically relaxed $c(2 \times 2)$ overlayer (the overlayer is compressed in the [110] direction to place 7 molecules over 12 copper atoms and expanded in the [001] direction to place 7 molecules over 16 copper atoms). (c) STM image showing the Moiré pattern observed at room temperature. (d) Simulated Moiré pattern resulting from a simplehard-ball stacking model of the structure in (b). This modification results in an almost hexagonal overlayer with $(12 \times 16)$ periodicity.

demonstrated, ${ }^{30}$ a stress rel ease pattern being the reason for a periodic substrate surface relaxation seems unlikely in the present case, since no evidencefor such a modulation was found even for the stronger bond sulfur in the bare $\mathrm{p}(5 \times 2)$ structure after thermal desorption of the alkyl chains. A primitive $\mathrm{c}(2 \times 2)$ structure on the $\mathrm{Cu}(110)$ surface (i.e., with one molecule per unit cell) yields a mol ecular packing area of $18.4 \AA^{2}$ per molecule, very close to the molecular packing area of $18.6 \AA^{2}$ found in bulk alkanethiols. A closer inspection reveals, however, very anisotropic nearest neighbor distances. While the molecules are separated by $5.1 \AA$ al ong the [1 10$]$ direction, the separation is only $4.4 \AA$ along [111] compared to a spacing of $4.6 \AA$ in a hexagonal close-packed configuration. Thus, a driving force for small relaxations in the $c(2 \times 2)$ structure could be a tendency of the alkane chains to maintain an isotropic intermolecular packing distance close to the bulk value. U sing a simple hard-ball stacking model, we have explored the coincidence meshes for a large variety of modified $\mathrm{c}(2 \times 2)$ overlayers on an unperturbed (110) substrate, resulting in Moiré periodicities with, or close to, the experimentally observed c(12 $\times 16)$ unit cell. The best agreement with the STM data is found for the model displayed in Figure 9b, obtained by anisotropic relaxation of a c $(2 \times 2)$ overlayer involving a $14 \%$ compression in the [110] direction and a $14 \%$ expansion in the [001] direction. These relaxations yield an almost hexagonal overlayer with a molecular packing area of $18.0 \AA^{2}$. This model clearly reproduces the protrusions (Figure 9d) indicated in the STM image (Figure 9c) but in addition features a number of slightly smaller protrusions along the [110] direction. These additional protrusions in the model are not seen in the STM image, but interconnections seem to exist between the experimentally observed protrusions al ong the [1]10] direction, while no interconnections are present for other high-symmetry directions in the STM image. These

(30) Grossmann, A.; Erley, W.; I bach, H. Surf. Sci. 1995, 337, 183. 
interconnections are even more pronounced after annealing, as shown in F igure 5. The lack of complete agreement between the STM image and a si mple hard-ball stacking model on an unperturbed substrate suggests that the observed Moiré pattern may result from a more complicated mechanism, perhaps also involving a lateral relaxation of the Cu substrate, similar to that observed for a sulfate-covered $\mathrm{Cu}(111)$ surface. ${ }^{31}$

No evidence for a pronounced reconstruction of the $\mathrm{Cu}(110)$ substrateunderneath thedodecanethiol layer was found in the present STM and LEED data. This is in contrast to the complete rearrangement of the top layer that has been observed foll owing the adsorption of thiols on $\mathrm{Cu}(111) .11,20$ The reconstruction on $\mathrm{Cu}(111)$ has been attributed to thetendency of sulfur atoms to occupy 4-fold coordinated sites. Theobserved stability of therather open $\mathrm{Cu}(110)$ surfaceis likely to be explained by theavailability of such specific adsorption sites in combination with the close agreement between the molecular packing area in the $\beta$ structureand in bulk alkanethiols. Whilethesurface terraces do not appear to reconstruct, significant step faceting occurred upon formation of the saturation structure as seen from Figure 3.

As mentioned earlier, indications exist that the $\alpha$ phase is a molecular bilayer. First, high-resolution STM images, like the one shown in Figure $4 \mathrm{~b}$, directly reveal features which appear to be holes within the $\alpha$ phase, exhibiting a c $(2 \times 8)$ superstructure $(\gamma$ phase). Second, the existence of a bilayer is corroborated by consideration of the molecular densities of the different structures. The areas per molecule in the $\alpha$ and $\gamma$ phases are 92.1 and $73.6 \AA^{2}$, respectively, if one/two dodecanethiol molecules for each $\left(\begin{array}{cc} \pm 8 & -2 \\ 1 & 1\end{array}\right) / c(2 \times 8)$ unit cell is assumed, as seems reasonable from the dimensions of the dodecanethiol molecules tentatively superposed on the corresponding STM images. Thec $(2 \times 2)$ saturation structure has a considerably higher molecular packing density with one upright mol ecule per $18.4 \AA^{2}$. Theobserved local reversibility of the $\alpha$ to $\beta$ phase

(31) Broekmann, P.; Wilms, M.; Wandelt, K. Surf. Rev. Lett. 1999, 6, 907. transition is facilitated if extensive mass transport is not required. Although the combined density of the $\alpha$ and $\gamma$ structures still does not fully suffice to provide the mol ecular density in the $\beta$ structure, theseconsi derations support the conclusion that the $\alpha$ phase is a molecular double layer.

\section{Conclusions}

The adsorption of dodecanethiol on a $\mathrm{Cu}(110)$ surface has been studied by STM, LEED, XPS, and TDS with a particular emphasis on the transient structures that appear upon formation of a saturated thiol atemonol ayer.

At the lowest temperature of $110 \mathrm{~K}$, an ordered layer of physisorbed molecules is observed. By choosing a fairly long chained alkanethiol, the physisorption energy becomes solargethat thetransition into chemisorbed species, that is, thiolateformation, sets in before desorption of the physisorbed species occurs. This allows a number of inter mediateadsor ption structures to beobserved at room temperature. Two intermediate pinstripe structures are observed, and indications exist that they are both part of a mol ecular doublelayer. Thesestructures transform into an approximate $c(2 \times 2)$ saturation phase. This closepacked structure reveals a long-range $c(12 \times 16)$ modulation. The saturated thiolate film is thermally metastable since partial dissociative desorption of about $25 \%$ of the alkyl chains has been observed after several hours. The resulting saturation structure consists of a coexisting thiolate and sulfide species. Heating the saturated film above $400 \mathrm{~K}$ causes a complete desorption of the alkyl chains, resulting in a $p(5 \times 2)$ sulfur structure.

Acknowledgment. This work has been supported by the Danish Natural Science Research Council and the Danish National Research F oundation through theCenter for Atomic-scale Materials Physics, as well as the German Science Foundation DFG (Contract WO 464/11-3). The authors acknowledgevaluablehel p from Kurt Gothelf and Erik Lægsgaard.

\author{
LA025661]
}

